\title{
Análisis, síntesis y evaluación de la literatura de lagartos de la Región de Atacama, Chile
}

\author{
Analysis, synthesis and evaluation of the literature of lizards from Atacama Region, Chile
}

Pablo Valladares Faúndez ${ }^{* \neq}$

*Unidad de Pasivos Ambientales y Ecosistemas, Centro Regional de Investigación y Desarrollo Sustentable de Atacama (CRIDESAT), Universidad de Atacama. Copayapu 485, Copiapó.

‡Departamento de Biología, Facultad de Ciencias, Universidad de Tarapacá. Velásquez 1775, Arica.

Email: pablo.valladares@cridesat.cl

\section{RESUMEN}

La herpetología ha sido una de las disciplinas científicas más estudiadas de la zoología atacameña, sin embargo su conocimiento es aún insuficiente. Por ejemplo no hay consenso en el número de especies que habitan esta región. Algunos autores estiman que existen alrededor de 18 especies del género Liolaemus, dos subespecies del teiido Callopistes maculatus y una especie de gecko del género Homonota. El presente trabajo tiene como objetivo realizar un análisis crítico de la literatura científica de los lagartos atacameños. Se analizaron las temáticas más desarrolladas, el nivel de conocimiento de las especies y los vacíos que aún existen. Revisamos los rangos de distribución según la literatura y algunas de las colecciones taxonómicas disponibles. Los resultados indican que se necesita revisar la taxonomía de varias de las especies que se asignan para la Región de Atacama. El nivel de exploración de la región es amplio, aunque aún falta realizar más colecciones taxonómicas para poder determinar los rangos de distribución, riquezas de especie por áreas específicas, abundancia y variación morfológica y genética. Gran parte de los estudios están restringidos a la taxonomía, pero pocos relacionados a la ecología y conservación. Las dudas taxonómicas son profundas, sobre todo las relacionadas a las poblaciones del grupo "nigromaculatus". Las dos subespecies de Callopistes maculatus descritas para Atacama no han vuelto a ser revisadas, al igual que la especie de gecko que habita la región. La especie mejor estudiada es Microlophus atacamensis, con estudios en ecología, genética, evolución y morfología. Existen ejemplares tipo extraviados, lo que dificulta el análisis de la situación taxonómica de algunos taxa.

Palabras clave: Lagartos de Atacama, biodiversidad, endemismo, riqueza taxonómica.

\begin{abstract}
Herpetology has been one of the most studied disciplines of zoology in the Atacama region, but our knowledge remains woefully incomplete. For example, there is no consensus on the number of species inhabiting this region. Some authors estimate 18 Liolaemus species, two subspecies of Callopistes maculatus and one species of geckkonid lizard. Our aim is to make a critical analysis of the scientific literature on lizards from the Atacama Region. We analyze the major issues, taxonomy of species recognized in this region and knowledge gaps that exist. We reviewed the distributional ranges for each species according to available literature and taxonomic sources. Our results indicate that many Atacama species require significant taxonomic revision. Although the region has been widely explored, additional taxonomic collections are essential to accurately determine the ranges, species richness in specific areas, abundance and morphological and genetic variation of almost all species. Many previous studies were restricted to taxonomy, and very few focused on ecology and conservation. There are extensive taxonomic uncertainties in many groups, especially those related to the "nigromaculatus" group. Two subspecies of Callopistes maculatus and the gecko species require significant revision; while Microlophus atacamensis is the most studied species in terms of ecology, genetics, evolution and morphology. Many type specimens are lost, making difficult the taxonomic analysis of some species.
\end{abstract}

KEYwORDS: Atacama lizards, biodiversity, endemism, taxonomic richness. 


\section{INTRODUCCIÓN}

La necesidad de hacer catastros de especies por regiones y análisis del estado del conocimiento de la biodiversidad ha sido muy importante en los últimos años en el contexto del cambio climático global, principalmente en lo relacionado a la caracterización de la riqueza específica local, niveles de endemismo y grado de amenazas (Elgueta et al. 2006). Esta práctica es importante para determinar los "hotspot" de biodiversidad (Myers et al. 2000) y determinar así las áreas de interés de conservación (Simonetti, 1999). Por otro lado, los análisis de catastros de biodiversidad permiten identificar, registrar y monitorear estacional y/o anualmente a los taxa (Rau, 2005), así como visualizar las áreas temáticas que se han desarrollado, las especies que se han estudiado y las áreas geográficas que aún quedan por explorar. Sin embargo, Chile aún mantiene grandes vacíos de información de estas temáticas, y particularmente en los ecosistemas áridos.

El caso de Atacama es muy singular, pues según el Comité Nacional de Biodiversidad (en Simonetti et al. 1995) el nivel de conocimiento de su biodiversidad es uno de los menos conocidos de Chile, situación que al menos en los vertebrados terrestres no ha sido revertida.

Las primeras observaciones realizadas en la Región de Atacama fueron hechas por Rudolph Amandus Philippi entre 1853 y 1854, pero publicadas en 1860 en su tratado "Viage al Desierto de Atacama" donde describe nueve especies de lagartos, sin dar mayores detalles de sus localidades específicas, lo que genera cierta confusión taxonómica. Recién en el siglo XX se comienzan a realizar catastros más exhaustivos de las especies de reptiles atacameños. Como resultado de ello se comienzan a describir una serie de especies y subespecies, como Liolaemus nigromaculatus atacamensis y L. n. copiapoensis (Müller \& Hellmich, 1933), Callopistes maculatus atacamensis Donoso-Barros, 1960a, Alsophis chamissonis erenicola (Donoso-Barros, 1974), L. velosoi Ortiz, 1987, L. silvai Ortiz, 1989, L. juanortizi Young-Downey \& Moreno, 1991, L. rosenmanni Núñez \& Navarro, 1992, L. patriciaiturrae y L. isabelae Navarro \& Núñez, 1993, L. nigroventrolateralis Ortiz, 1994 (= L. isabelae), L. josephorum Núñez, Schulte \& Garín, 2001 y Phrynosaura manueli [sic] (Núñez, Navarro, Garín, Pincheira-Donoso \& Meriggio, 2003). Sólo en la última década se comienzan a realizar estudios de Ecología, Genética y Evolución (Benavides et al. 2007, Sepúlveda et al. 2008, Victoriano et al. 2003, Vidal \& Ortiz 2004a, 2004b, Vidal et al. 2002).

Sin embargo, las dudas taxonómicas en varias de las especies que se asignan a esta región siguen sin resolverse, aún no tenemos certeza de cuántas especies habitan esta región, ni cuál es la distribución y abundancia de ellas, por lo que el objetivo del presente trabajo es sintetizar, analizar y evaluar la información científica concerniente a las especies de lagartos descritos para Atacama y así identificar los vacíos de conocimiento para desarrollar una estrategia de investigación que revierta esta situación.

\section{MATERIAL Y MÉTODOS}

Para hacer un análisis del nivel de conocimiento de la herpetofauna atacameña, se tomó como referencia el listado de trabajos científicos publicados en diversas revistas científicas nacionales e internacionales que se encuentran citadas en la página web "Recopilación de la Literatura Herpetológica Chilena desde 1780 hasta 2010" (Silva-Aránguiz 2001), la cual se encuentra administrada por el Centro de Investigación Avanzada en Ecología y Biodiversidad (CASEB), del Departamento de Ecología de la Pontificia Universidad Católica de Chile. En una primera instancia se sintetizó la información del siglo XIX como una reseña histórica. Posteriormente, se identificaron los trabajos desde 1900 hasta la fecha que mencionaban o utilizaban como objeto de estudio a especies de lagartos presentes en la Región de Atacama, excluyendo libros, tesis, guías de campo e informes técnicos. Se actualizó el listado realizando una búsqueda de trabajos publicados recientemente en motores de búsqueda como ISI Web of Knowledge. Además se revisaron revistas de ciencias naturales chilenas como Medio Ambiente, Gayana, Boletín del Museo Nacional de Historia Natural de Chile, Noticiario Mensual del Museo Nacional de Historia Natural de Chile, Publicaciones Ocasionales del Museo Nacional de Historia Natural de Chile y Revista Chilena de Historia Natural. A partir del análisis de los trabajos recopilados se evaluaron las temáticas científicas mayormente abordadas, además de la identificación de las zonas de recolecta, información que fue complementada con los datos existentes en las colecciones taxonómicas del Museo Nacional de Historia Natural de Chile (MNHN) y del Museo de Zoología de la Universidad de Concepción (MZUC). Para efectos de la estimación de la riqueza de especies y rangos de distribución en la región se consideraron además los textos de Donoso-Barros (1966), Veloso \& Navarro (1988), Pincheira-Donoso \& Núñez (2005) y Vidal \& Labra (2008).

\section{RESULTADOS}

1. ANÁLiSiS DE LA INFORMACIÓN BIBLIOGRÁFICA DE LAGARTOS DE LA Región De ATACAMA.

Publicaciones ANTES de 1900

La descripción de especies de lagartos de la Región de Atacama, antes del siglo XX, tiene la particularidad que en 
su mayoría fueron descritas para otras regiones del país y que posteriormente, durante el siglo XX, fueron asignadas a esta región. Por ejemplo L. andinus Kolowsky, 1895, especie que fue originalmente descrita para la región de Catamarca, Argentina, y ha sido asignada para nuestro país por Halloy et al. (1991). Similar situación es la de $L$. fuscus Boulanger, 1885, especie pequeña y muy abundante en la zona central de Chile. L. nitidus (Wiegmann, 1835) y L. platei Werner, 1898 fueron descritas gracias a las recolectas de los naturalistas alemanes Meyen y Plate respectivamente. Sin embargo, ninguno de estos naturalistas visitó la Región de Atacama. Sólo Philippi entre 1853 y 1854 recorrió la Región desde el puerto de Caldera hacia el norte, describiendo especies de las cuales sólo una parte de los ejemplares recolectados se encuentran depositados en el Museo Nacional de Historia Natural de Chile (Ortiz \& Núñez 1986). Otra espcie descrita en el siglo XIX fue Proctotretus nigromaculatus Duméril \& Bibron, 1837, la que posteriormente fue asignada a un grupo de subespecies con distribución entre las regiones de Antofagasta y Coquimbo (Donoso Barros, 1966). Además, Duméril \& Bibron (1839) describieron otras dos especies de lagartos que habitan la Región de Atacama, Gymnodactylus gaudichaudi ( $=$ Homonota gaudichaudi) y Aporomera ornata (= Callopistes maculatus), especies que fueron revisadas y descritas en el Museo Nacional de Historia Natural de París por Gaudichaud y D'Orbigny (Ortiz 2008), y que correspondieron a ejemplares colectados por Gay (en Gay, 1848).

PublicaCiones DESPuÉs DE 1900

Se identificaron 391 publicaciones de reptiles de Chile desde 1900 hasta la fecha, de las más diversas temáticas. De ellos, sólo 42 analizaron alguna variable biológica de lagartos estudiados en la Región de Atacama, lo que representa un $10,51 \%$ del total de publicaciones. La mayor parte de los artículos analizadas tratan más de una temática científica a la vez. El mayor número de publicaciones se encuentra relacionado a la taxonomía y sistemática de las especies $(65,6 \%)$. Las temáticas tratadas secundariamente son Ecología (23,6\%), Morfología y Fisiología $(23,1 \%$ ), Distribución $(23,1 \%)$ y Evolución y Biogeografía $(19,1 \%)$. Las áreas menos tratadas son Genética (7,7\%), Conducta, Reproducción y Conservación (cada una con un 5,1\%). No se ha publicado ningún trabajo en las temáticas de enfermedades y parásitos de lagartos presentes en la región.

El 16\% de los trabajos analizados miden, evalúan, analizan o sencillamente mencionan alguna variable biológica de Microlophus atacamensis (Donoso-Barros, 1960c), convirtiéndose en la especie más estudiada de la región.
La siguen C. maculatus (8,5\%), H. gaudichaudi $(6,4 \%)$, L. atacamensis, L. manueli, L. patriciaiturrae y L. platei con $5,3 \%$ cada una. L. bisignatus, $L$. copiapoensis, $L$. nigromaculatus y $L$. rosenmanni con $4,3 \%$; $L$. isabelae, $L$. josephorum, L. juanortizi, L. nigroventrolateralis, L. nitidus, L. silvai y $L$. velosoi con un $3,2 \%$; L. andinus, L. eleodori Cei, Etheridge \& Videla, 1983, L. fuscus con 2,1\%; L. melanopleurus y L. nigriceps (Philippi, 1860) con 1,1\% de menciones, y L. lorenzmulleri Hellmich, 1950, L. robertoi Pincheira-Donoso \& Núñez, 2003 y L. zapallarensis (Müller $\&$ Hellmich, 1933) sin menciones.

Los estudios en M. atacamensis van desde lo taxonómico (Ortiz 1977, 1980; Ortiz \& Serey 1979), evolutivo (Benavides et al. 2007 Victoriano et al. 2003), fisiológico, morfológico y reproductivo (Sepúlveda et al. 2008 Vidal \& Ortiz 2004a) y ecológico (Fariña et al. 2008 Vidal et al. 2002 DonosoBarros 1960b). En relación a C. maculatus, las temáticas están relacionadas a lo taxonómico y distribución (DonosoBarros, 1960a, 1960b) y dieta (Vidal \& Ortiz 2004b). En $H$. gaudichaudi, los datos más recurrentes son de distribución (Donoso-Barros 1960b; Capurro 1957). Para las especies del género Liolaemus, el 68\% de las publicaciones abordan temáticas taxonómicas.

\section{EsPeCies adscritas a la Región de ATACAMA}

De acuerdo a distintos autores, se han mencionado para la Región de Atacama cuatro géneros de lagartos. Liolaemus es el que cuenta con mayor representatividad con 22 especies, Callopistes con dos subespecies, Microlophus y Homonota con una especie cada una (Tabla 1). Las especies de lagartos que se asignan para la Región de Atacama se mencionan a continuación:

L. andinus Koslowsky, 1895, es mencionada por primera vez para la Región de Atacama por Halloy et al. (1991). Esta autora afirma que la subespecie L. a. andinus se encontraría al oriente del salar de Maricunga, en una localidad denominada Cuesta Colorada, en el paso San Francisco. Agrega que las poblaciones al lado oriental de los Andes corresponderían a L. andinus poecilochromus (Laurent, 1982). Esta especie habitaría los Andes altiplánicos de Argentina, Chile y Bolivia. Particularmente en Chile habitaría los alrededores de San Pedro de Atacama hacia el este, en la Segunda Región de Antofagasta (Pincheira-Donoso \& Núñez 2005) y al sur por el paso de San Francisco, en la Tercera Región de Atacama (Halloy et al. 1991). También esta especie es mencionada para Atacama por Díaz-Páez et al. (2008). Según Schulte et al (2000) y Lobos et al. (2010) pertenece a la serie "montanus" de la sección "montanus". Los ejemplares sintipo estaban depositados en el Museo de la Plata, Argentina, pero ahora se encuentran extraviados (Etheridge \& Frost 2010). 


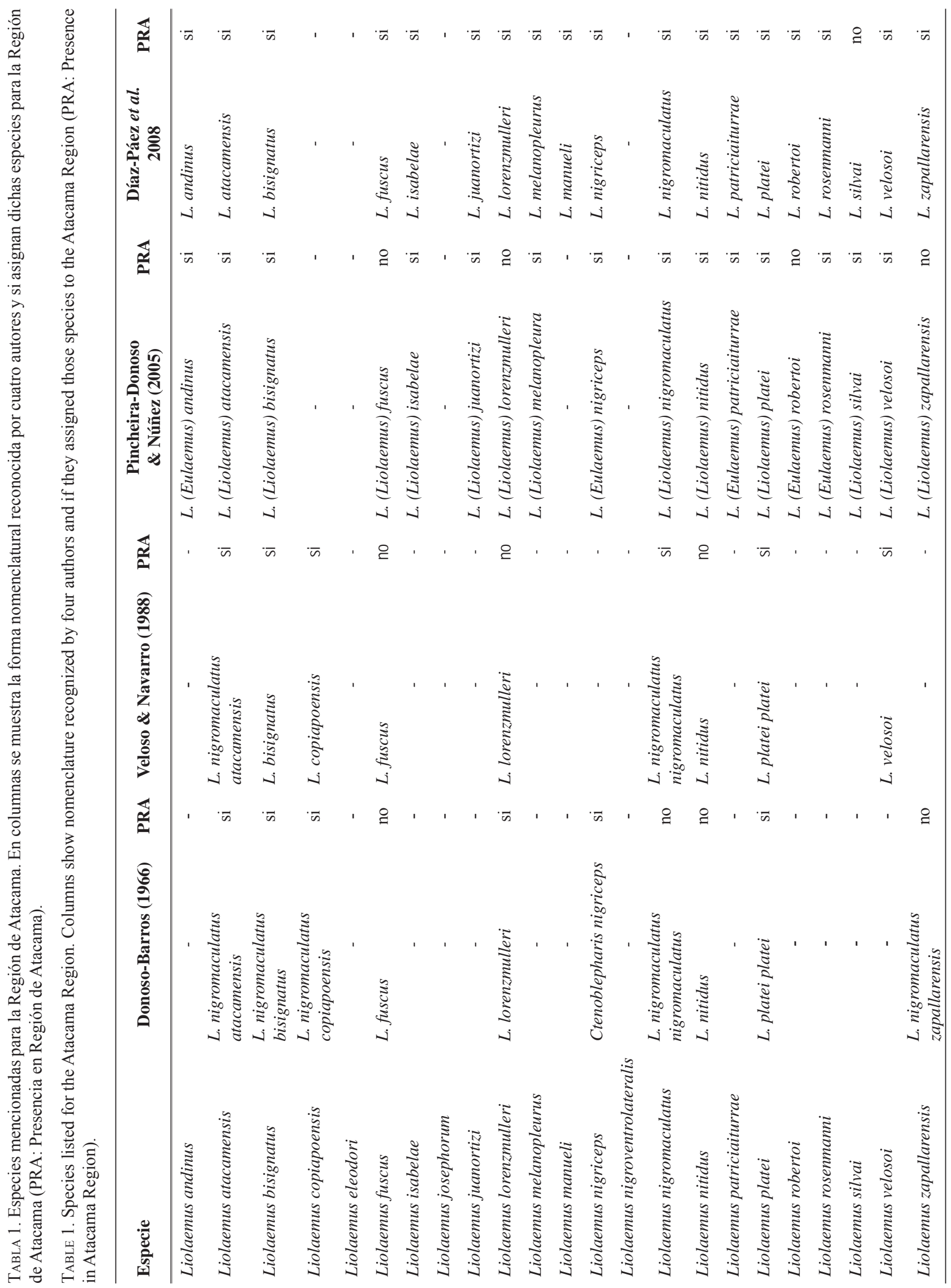


L. atacamensis Müller \& Hellmich, 1933, es una lagartija característica de la Región de Atacama. Su taxonomía ha sido controversial, dado que pertenece a uno de los grupos más confusos de la herpetología chilena. Fue descrita inicialmente como una subespecie de L. nigromaculatus, criterio que fue seguido por varios autores (Laurent 1992, Veloso \& Navarro 1988, Donoso-Barros, 1966, 1970), hasta que Núñez \& Jaksic (1992) la elevan al estatus de especie, acción que es confirmada por Etheridge (1995). Según Hellmich (1952) habita "el desierto de rodados de Atacama", su terra typica es "Atacama, nordöstlich von Copiapó, Nordchile" es decir, al noreste de Copiapó (Müller $\&$ Hellmich 1933). Presenta un alto nivel de polimorfismo en su morfología, el que tiene relación con el tamaño de los ejemplares, el número de escamas alrededor del cuerpo, así como las coloraciones y diseños en el dorso. Tiene una amplia distribución geográfica, que sería desde el norte de Copiapó hasta Illapel, por el valle central (Donoso Barros, 1966), o desde el sur de Antofagasta, como Taltal, hasta el norte de la Región de Coquimbo (Pincheira-Donoso \& Núñez 2005) alcanzando los $3000 \mathrm{~m}$ de altitud (Veloso \& Navarro 1988). Es clasificado dentro del subgénero Liolaemus, en el grupo "nigromaculatus" (Lobos et al. 2010).

L. bisignatus (Philippi, 1860) es un lagarto de mayor tamaño que L. atacamensis. Su descripción original es acotada, con una breve descripción de sus escamas dorsales y no detalla el lugar de captura. Sin embargo, Müller \& Hellmich (1933) la reconocen como subespecie de L. nigromaculatus, al igual que Donoso-Barros $(1966,1970)$ y es considerada como una buena especie por Ortiz (1981) y Veloso \& Navarro (1988). Müller \& Hellmich (1933) y Hellmich (1952) señalan su terra typica para los alrededores de Caldera, ya que tiene designada un ejemplar tipo en la Colección Herpetológica del Museo de Historia Natural de Chile (MNHN 1477, Ortiz \& Núñez, 1986), capturado por Philippi en la localidad de Caldera. Moreno et al. (2001) mencionan como localidades de captura seis ejemplares en El Faro, comuna de Caldera. Müller \& Hellmich (1933) designaron un neotipo para esta especie (Zoologischen Staalssamlung de München $\mathrm{N}^{\circ} 181 / 1921 \mathrm{a}$ ), el que ha sido considerado inválido por Etheridge \& Frost (2010) por la existencia previa del sintipo de Philippi (1860). Esta especie también es asignada al subgénero Liolaemus, en el grupo "nigromaculatus" (Lobos et al. 2010).

L. copiapoensis Müller \& Hellmich, 1933, fue diagnosticada de L. bisignatus por su menor tamaño, ausencia de tonos verdes (Hellmich 1952) y de quillas en las escamas temporales (Donoso-Barros, 1966). Esta especie es aceptada por Ortiz (1981), Veloso \& Navarro (1988), Núñez \& Jaksic (1992) y Etheridge (1995, como L. copiapensis), Vidal et al. (2008) y Etheridge \& Frost (2010). Previamente fue reconocida como subespecies de L. nigromaculatus (Donoso-Barros 1966, 1970, Müller \& Hellmich 1933). Sin embargo, esta especie es sinonimizada a $L$. bisignatus por Pincheira-Donoso \& Núñez (2005).

L. eleodori Cei, Etheridge \& Videla, 1983, es una especie descrita para la Reserva Provincial de San Guillermo en la Provincia de San Juan, Argentina. Esta especie fue adscrita a Chile para los Andes de la Región de Atacama por Núñez \& Torres-Mura (1992), los que capturaron siete ejemplares a

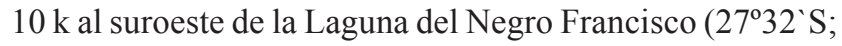
$69^{\circ} 19^{\circ} \mathrm{W}$ ) a $4.100 \mathrm{msnm}$. Según estos autores, es posible la existencia de esta especie en este lugar dado que su terra typica está ligeramente más al sur, en un ambiente muy similar. Sin embargo, Pincheira-Donoso \& Núñez (2005) afirman que estos ejemplares corresponden a machos de $L$. rosenmanni, criterio que siguen Vidal et al. (2008) y DíazPáez et al. (2008). Esta especie pertenece al subgénero Eulaemus, sección "montanus", serie "montanus" (Lobos et al. 2010).

L. fuscus Boulanger, 1885, es una especie pequeña, de aspecto frágil que se caracteriza por una banda negra vertebral, acompañada de dos bandas blancas laterales y paralelas (Donoso-Barros 1966). Es una especie con un amplio rango de distribución, que va desde el norte de Illapel hasta el sur de Concepción (Donoso-Barros 1966), aunque Veloso \& Navarro (1988) la restringen desde la Región Metropolitana hasta el Bio Bío. Aparece por primera vez como miembro de la fauna atacameña en Moreno et al. (2001), donde menciona tres ejemplares capturados en Huasco. Díaz-Páez et al. (2008) también la mencionan para la Región de Atacama, aunque no detallan localidades de captura. Su terra typica es Valparaíso; la descripción original estuvo basada en un ejemplar capturado de los cerros aledaños a dicho puerto. Pertenece al subgénero Liolaemus (Schulte et al. 2000), en el grupo "alticolor-bibronii" (Lobos et al. 2010).

L. isabelae Navarro \& Núñez, 1993, es una especie endémica de Atacama, los machos son profundamente melánicos con copiosas manchas amarillas en la cabeza, las patas y la cola, con dimorfismo sexual y muy polimórfica. Es una especie esbelta, de tamaño mediano. Su terra typica es "el cerrito", $12 \mathrm{~km}$ NO campamento La Ola $\left(26^{\circ} 27^{\prime} \mathrm{S} ; 6^{\circ} 03^{\prime} \mathrm{W}\right)$ a 3.556 msnm. Según Pincheira-Donoso \& Núñez (2005) esta especie habitaría en la mina "El Hueso", Potrerillos, 2.850 msnm, en la Quebrada de Pedernales, cerca del Salar de Pedernales a $3.672 \mathrm{msnm}$, y en el mismo Salar de Pedernales a $3.425 \mathrm{msnm}$. Especie reconocida por Etheridge \& Espinoza (2000), Pincheira-Donoso \& Núñez (2005), DíazPáez et al. (2008) y Etheridge \& Frost (2010). Perteneciente al subgénero Liolaemus, en el grupo "nigroviridis" (Lobos 2005, Lobos et al. 2010). 
L. josephorum Núñez, Schulte \& Garín, 2001, es un lagarto pequeño, afín a $L$. velosoi y L. platei. Su terra typica es Diego de Almagro (26²3'S; 7002'W; 750 msnm). Según Núñez et al. (2001), y de acuerdo a un análisis filogenético por medio del análisis de Máxima Parsimonia, esta especie estaría conformando un grupo monofilético con L. platei. Sin embargo, esta especie es sinonimizada a L. velosoi (Pincheira-Donoso \& Núñez, 2005). Se cuenta con registros de recolecta en los alrededores de Diego de Almagro, en sectores conocidos como Sierra de Santo Domingo, la Finca de Chañaral y El Salvador, a 3.500 msnm.

L. juanortizi Young-Downey \& Moreno, 1991, es un lagarto de tamaño moderado a grande, las hembras adultas y juveniles presentan un color ventral gris, y machos adultos negro brillante. Tono general de fondo café amarillento, con diseño dorsal de manchas oscuras en el centro de las escamas. Esta especie fue inicialmente capturada en Río Aguas Blancas

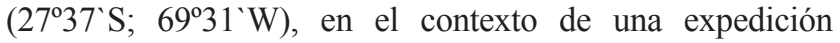
organizada por el Museo Regional de Atacama en 1988 (Young-Downey \& Moreno, 1991). Esta especie es omnívora, habita zonas con pequeños matorrales, aunque prefiere esconderse bajo piedras. Recientemente ha sido capturada en Aldebarán, Río La Gallina, donde vive en simpatría con $L$. nigriceps (Pincheira-Donoso \& Núñez, 2005). Moreno et al. 2001 mencionan para la Colección Herpetológica del Museo del Mar de la Universidad Arturo Prat un espécimen capturado en Quebradas Aguas Blancas, Copiapó, la que corresponde a su terra typica. Presenta un ejemplar holotipo, el que en un primer momento fue custodiado en el Museo Regional de Atacama (MRA 0139). Sin embargo, en la actualidad este ejemplar se encuentra perdido, ya que no se encuentra en dicho museo (Raúl Céspedes com. per.) ni en la colección de la Universidad de Concepción (Juan Carlos Ortiz com. per.). Según Lobos et al. (2010) es parte del subgénero Liolaemus, de la sección "montanus", serie "montanus".

L. lorenzmulleri Hellmich, 1950, es una especie de lagarto de tamaño grande, con escamas dorsales imbricadas. Tono de fondo en general café amarillento, con manchas oscuras en las escamas. Zona ventral blanquecina con manchas negras extendidas por toda la zona. Es una especie con una historia taxonómica estable, siendo reconocida por DonosoBarros (1966, 1970), Veloso \& Navarro (1988), Núñez \& Jaksic (1992), Etheridge \& Espinoza (2000) y Lobos et al. (2010) como parte del subgénero Liolaemus, en el grupo "nigroviridis". De acuerdo a la información recopilada en la literatura científica y colecciones taxonómicas, habita sólo en la Región de Coquimbo, en los alrededores de la Cordillera de Doña Ana, desde Baños del Toro por el norte, hasta el Embalse La Laguna al sur de Nueva Elqui. Sin embargo, Donoso-Barros (1966) y Díaz-Páez et al. (2008) la reconocen para la Región de Atacama sin dar antecedentes de capturas en esta Región.
L. manueli (Núñez, Navarro, Garín, Pincheira-Donoso \& Meriggio 2003) es un lagarto de tamaño pequeño. Inicialmente fue descrito bajo el género Phrynosaura (Werner en Bürger 1907). Esta especie fue denominada como P. cf audituvelata por Núñez et al. (1998) por parecer incierta su posición taxonómica. Esta especie es reconocida por Schulte et al. (2004), Valladares (2004) y Vidal et al. (2008). Lobos et al. (2010) la reconoce como parte de la sección "montanus", de la serie "montanus". Tiene como terra typica a la comuna de Diego de Almagro (26 $233^{\circ} \mathrm{S}$; $70^{\circ} 02^{`} \mathrm{~W}$ ) a una altura de $750 \mathrm{msnm}$, pero ha sido capturado $16 \mathrm{~km}$ al sur, lugar denominado "Finca de Chañaral". Esta especie es simpátrica con H. gaudichaudi, C. maculatus, L. josephorum y L. cf atacamensis. Habita terrenos arenosos, construyendo pequeñas cavidades como en lugares de baja productividad, se encuentra usualmente bajo piedras de tamaño variable, su abundancia es escasa.

L. melanopleurus (Philippi, 1860) es una de las especies descrita en el contexto del "Viaje al Desierto de Atacama" entre 1853 y 1854 . En este trabajo no detalla su lugar de captura ni un punto de referencia que pudiera realmente asignarlo a la Región de Atacama. Existen sólo tres especímenes asignados a esta especie, dos en el Museo de Historia Natural de Chile y otro en el Field Museum of Chicago. Ortiz \& Núñez (1986) afirman que se encontró un frasco con dos especímenes que estaban etiquetados como Liolaemus darwini Blg. Bajo esta etiqueta se encontró otras con la letra de Philippi, donde se pudo leer nítidamente "Pr. melanopleurus”. Según Pincheira-Donoso \& Núñez (2005), esta especie sería afín con L. platei, pero se diferencia de ésta por presentar mayor número de escamas alrededor del cuerpo y por la presencia de una franja lateral negra que se extiende desde la axila a la ingle, característica que efectivamente menciona Philippi (1860) en la descripción original. Esta especie es reconocida por Etheridge (1995), Ortiz (2008) y Vidal et al. (2008). Lobos et al. (2010) la reconoce como parte del grupo "nigroviridis". Sin embargo, solo se conoce el ejemplar sintipo, sin existir más colectas de esta especie.

L. nigriceps (Philippi, 1860) es una especie de tamaño mediano a grande, caracterizada por presentar un capuchón negro en la cabeza, cuello y garganta, capturada cerca de Pajonal, alrededor de los $3400 \mathrm{~m}$ sobre el nivel del mar, actualmente en la Región de Antofagasta. Fue considerada como una variedad de L. signifer Koslowsky, 1898 y como subespecie L. S. nigriceps (Burt \& Burt, 1933). Posteriormente fue adscrita al género Ctenoblepharys por Donoso-Barros (1966, 1970) y Veloso \& Navarro (1988). Laurent (1992) la asigna a Liolaemus, criterio seguido por Núñez \& Jaksic (1992), Etheridge (1995), Etheridge \& Frost (2010) y Lobos et al. (2010), este último como parte del subgénero Liolaemus, de la sección "montanus", serie 
“montanus". Su distribución es Río Frío y Quebrada Zorritas en el Volcán Llullaillaco, en la Región de Antofagasta. En Argentina ha sido documentada para la localidad de Socompa, en la Provincia de Salta y en Olacapato, Provincia de Jujuy y se menciona para la Región de Atacama en la zona de Aldebarán, en el Río La Gallina, al este de Copiapó (Pincheira-Donoso \& Núñez 2005). Esta especie habita lugares arenosos, con predominancia de biotopos pedregosos, usa cuevas de roedores u oquedades de rocas para protegerse. En la Colección Herpetológica del Museo de Zoología de la Universidad de Concepción, se encuentra una serie de ejemplares colectados por Juan Carlos Ortiz

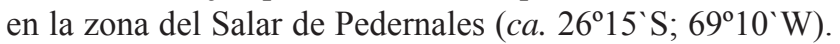
Según Ortiz \& Núñez (1986), de los dos sintipos colectados por Philippi, uno está perdido y el otro se encuentra en el Zoologischen Staalssamlung de München con el número 38/1930, el que le había sido facilitado a Walter Hellmich por Ernesto Gigoux, pero este ejemplar habría sido devuelto a su remitente.

L. nigroventrolateralis Ortiz, 1994, es una especie de tamaño mediano a grande, afín con $L$. nigriceps, pero se diferencia en que $L$. nigroventrolateralis presenta una línea media dorsal de color amarillo, es un poco más pequeño y delgado, y posee menor número de escamas alrededor del cuerpo. Su terra typica corresponde a las cercanías del Salar de Pedernales (26ำ $\left.15^{\prime} \mathrm{S} ; 69^{\circ} 10^{\prime} \mathrm{W}\right)$, a $3.425 \mathrm{msnm}$, Provincia de Chañaral, Región de Atacama. Sin embargo, esta especie fue sinonimizada por Pincheira-Donoso \& Núñez (2007) ya que los caracteres diagnósticos identificados por Ortiz (1994) corresponden con los previamente definidos para L. isabelae (Navarro \& Núñez 1993). La cantidad de caracteres morfológicos y morfométricos similares parecen efectivamente indicar la sinonimia.

L. nigromaculatus (Wiegmann 1835), es un lagarto de tamaño mediano, que habita las costas de la Región de Atacama, donde existen zonas arenosas, pero prefiere esconderse en zonas pedregosas (Gigoux 1927), aunque es muy hábil para enterrarse en la arena si la situación lo amerita. Es de hábitos psamófilos (Donoso-Barros 1966). Según Hellmich (1952) y Donoso-Barros (1970) su terra typica es Huasco. Esta especie tiene una larga historia taxonómica, y por lo tanto muy confusa. Inicialmente fue descrita en el género Tropidurus (Wiegmann, 1835), siendo después adscrita a los géneros Proctotretus Duméril \& Bibron, 1837, Ptychodeira Fitzinger, 1843, Rhytidodeira Girard, 1857, hasta que Boulenger (1885) lo asigna correctamente a Liolaemus. Por otro lado, Müller \& Hellmich (1933) designan varias subespecies, entre ellas L. n. nigromaculatus, L. n. zapallarensis, L. n. atacamensis y L. n. copiapoensis. Lobos et al. (2010) la incorporan al subgénero Liolaemus, grupo "nigromaculatus".
L. nitidus (Wiegman, 1834) es una especie de tamaño grande, cuerpo robusto, escamas dorsales de gran tamaño, fuertemente carenadas, quilladas e imbricadas. Su colorido es muy variable, pero en general sus escamas son oscuras, grises o verde oscuro, con bandas oscuras irregulares dirigidas anteroposteriormente (Donoso-Barros 1966). Su taxonomía es estable a partir de Burt \& Burt (1930), sin presentar cambios sustanciales, ni a nivel genérico, específico ni subespecífico. Tiene un amplio rango de distribución, desde El Parque Nacional Llanos de Challe por el norte, Región de Atacama (Moreno et al. 2000, 2002) hasta el sur de Concepción (Donoso-Barros 1966). Moreno et al. (2001) menciona a cuatro ejemplares capturados en Vallenar. Según Lobos (2005) y Lobos et al. (2010) pertenece al grupo "robertmertensi".

L. patriciaiturrae Navarro \& Núñez, 1993, es un lagarto de mayor tamaño que $L$. isabelae, con la que coexiste. Pertenece a la sección "montanus", serie "montanus" (Lobos et al. 2010). Se refugia entre las piedras resquebrajadas. Al parecer, es endémica de la Región de Atacama, de alimentación mixta (insectos y vegetales). Es una especie ampliamente reconocida (Etheridge 1995, Etheridge \& Espinoza 2000 y Pincheira-Donoso \& Núñez 2005). Según estos últimos autores, la especie se distribuiría en la mina "El Hueso" en Potrerillos, al sur-este de El Salvador, alrededores del Salar de Pedernales y La Ola, siendo ésta última su terra typica. Moreno et al. (2001) menciona un individuo capturado en Nevado Jotabeche, Copiapó.

L. platei Werner, 1898, es una especie pequeña, de aspecto frágil. Se caracteriza por presentar escamas dorsales fuertemente imbricadas, el color de fondo del dorso es grisáceo azulado, con manchas transversales negras e irregulares y flancos con grandes manchas negras, también irregulares. Algunos ejemplares presentan un capuchón amarillo. Su historia taxonómica es muy regular, es una especie aceptada ampliamente por los herpetólogos chilenos y extranjeros. Presenta una amplia distribución, desde Paposo por el norte hasta Coquimbo (Ortiz 1973), siendo esta última su terra typica. En la Provincia de Copiapó ha sido colectada en El Pretil, Cerro La Diuca, Totoral, Los Loros y Caldera (El Faro), Quebrada El León y en la Provincia de Huasco en la localidad de San Félix (Moreno et al. 2001, Ortiz 1973). Es parte del subgénero Liolaemus (Schulte et al. 2000), del grupo “nigromaculatus” Lobos et al. (2010).

L. robertoi Pincheira-Donoso \& Núñez, 2003, es un lagarto de tamaño mediano, de apariencia robusta. Inicialmente esta especie fue asignada a L. cf vallecurensis (Núñez \& TorresMura 1992, Cortés et al. 1995). Sin embargo, un análisis posterior determinó que esta especie era un taxa no descrito para la ciencia (Pincheira-Donoso \& Núñez 2003). Su terra typica es Estero Tambo, Piedra Colgada, El Indio (2948 ' S; 
$\left.69^{\circ} 58^{\prime} \mathrm{W}\right)$, a $3.700 \mathrm{msnm}$, en la Región de Coquimbo. Alotipo capturada en la localidad de Río Toro Muerto, el Indio, a $2.400 \mathrm{msnm}\left(29^{\circ} 13^{`} \mathrm{~S} ; 70^{\circ} 33^{\circ} \mathrm{W}\right)$, paratipos en Baños del Toro, Sector Alto, todos en la misma región. Habita zonas caracterizadas por pequeños roquedales de poca pendiente. Fue asignada a la Región de Atacama por Díaz-Páez et al. (2008) sin dar más antecedentes. Pertenece a la sección "montanus", serie "montanus" (Lobos et al. 2010).

L. rosenmanni Núñez y Navarro, 1992, es una especie robusta, de tamaño mediano, presenta manchas dorsales irregulares negras, los machos presentan garganta y vientre melánicos. Sus escamas dorsales son lisas, redondeadas y yuxtapuestas, entre ellas escamas pequeñas. Esta especie habita la alta cordillera de Copiapó, su terra typica es Pastos Largos, Chimberos, Copiapó (265ㄴ 'S; 6955`W) 1964 msnm. También ha sido colectada en el Salar de Maricunga (26 $55^{\circ}$ S; $69^{\circ} 05^{`} \mathrm{~W}$ ) a $3.750 \mathrm{msnm}$, Quebrada Campamento, mina La Coipa $\left(26^{\circ} 56^{`} \mathrm{~S} ; 96^{\circ} 15^{`} \mathrm{~W}\right)$ a $3.750 \mathrm{msnm}$. Según Pincheira-Donoso \& Núñez (2005), esta especie se distribuye desde el Salar de Pedernales por el norte hasta Pastos Largos, Chimberos, por el sur. Los ejemplares designados a L. eleodori por Núñez \& Torres-Mura (1992) de la Laguna del Negro Francisco corresponderían al macho de L. rosenmanni (Pincheira-Donoso \& Núñez 2005). Pertenece a la sección "montanus", serie "montanus" (Lobos et al. 2010).

L. silvai Ortiz, 1989 es una especie de tamaño mediano, adscrita al grupo "nigromaculatus" (Lobos et al. 2010). Se caracteriza por su diseño dorsal muy conspicuo, con 9 a 11 barras negras transversales, con mancha antehumeral muy intensa, alargada, alcanzando el pliegue prehumeral, presenta una constricción medial que le confiere un aspecto de reloj de arena (Pincheira-Donoso \& Núñez 2005). Es una especie sin mayores controversias taxonómicas, reconocida por Núñez \& Jaksic (1992), Etheridge \& Espinoza (2000), Pincheira-Donoso \& Núñez (2005) y Vidal et al. (2008).

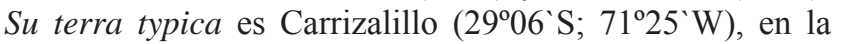
Provincia de Huasco, Región de Atacama (Ortiz 1989). Es conocida sólo en su localidad tipo, la que corresponde a un biotopo costero, en sectores rocosos. Según PincheiraDonoso \& Núñez (2005) vive en simpatría con C. maculatus, H. gaudichaudii y los colúbridos Philodryas chamissonis y Tachymenis chilensis. Es de hábitos insectívoros.

L. velosoi Ortiz, 1987 (L. velosi Núñez et al. 2001, nomen enmendatum), es una especie de tamaño pequeña, esbelta, que se diferencia de L. platei en el ordenamiento de las escamas del borde anterior de la abertura auditiva, que corresponden a dos series de pequeñas escamas que la recubren parcialmente. Su terra typica es desvío al Cerro Imán, al noroeste de la ciudad de Copiapó, Región de Atacama. Los especímenes paratipos fueron capturados en la Estación Paipote y Piedra Colgada, también en los alrededores de Copiapó. Además, hemos podido colectar especímenes al interior de la Universidad de Atacama en simpatría con L. platei. Su distribución está ligada al lecho del río Copiapó, caracterizados por matorrales de Geoffroea decorticans, Acacia cavens y Baccharis sp. Es una especie insectívora y endémica de la Región de Atacama (Ortiz 1987). Esta especie es de tono general grisáceo blanquecino o gris amarillento muy pálido, sobre el cual se dispone un diseño de manchas negras ordenadas en series transversales, flancos con manchas melánicas. Se diferenciaría de $L$. platei porque esta última posee mayor número de escamas alrededor del cuerpo, sus escamas dorsales más fuertemente quilladas y por un color parduzco, con flancos más melánicos y salpicados de matices celestes. Lobos et al. (2010) la adscribe al grupo "nigromaculatus".

L. zapallarensis Müller \& Hellmich, 1933, es un lagarto de gran tamaño, de apariencia robusta, dorso de color oscuro, generalmente negro Ortiz (1981) reconoce tres subespecies, L. z. zapallarensis, con algunas manchas dorsolaterales claras, vientre blanquecino, muy contrastante al color dorsal, su terra typica es Zapallar, Región de Valparaíso; L. z. ater, que es endémica de la Isla de Pájaros, frente a Coquimbo, y que es negra completa, ventral y dorsalmente. La otra subespecie es L. Z. sieversi, que habita exclusivamente en la Isla de Locos, frente a Pichidangui, esta carece de bandas dorsolaterales blancas, en su lugar tiene manchas amarillentas en los costados de la espalda. La historia taxonómica de este grupo ha sido larga y controversial, siendo éstas asignadas a su vez como subespecies de $L$. nigromaculatus (Donoso-Barros, 1966, 1970, Müller \& Hellmich, 1933). Es una especie que habita desde la Región de Coquimbo por el norte, hasta Valparaíso por el sur, encontrándose en Fray Jorge, Coquimbo, Isla de Pájaros y de Locos, Los Vilos, Pichidangui, etc. Sin embargo, es mencionada para la Región de Atacama por Díaz-Páez et al. (2008) sin dar más antecedentes. Es asignada al grupo “nigromaculatus" (Lobos et al. 2010).

M. atacamensis Donoso-Barros, 1960c, es un lagarto de tamaño grande, de color negro petróleo, región ventral con tintes azulinos. Vive en los rompeolas de las costas de Atacama. Su terra typica es Bahía Inglesa, Comuna de Caldera (Donoso-Barros, 1960c, 1966). Inicialmente fue adscrita al género Tropidurus y como subespecie de peruvianus. Fue nuevamente asignada al género Microlophus por Frost (1992), del cuál era su género original (Duméril \& Bibron 1837). M. atacamensis es una de las especies del género asociadas al borde costero, similar a $M$. quadrivittatus. Estas dos especies fueron diferenciadas por caracteres morfológicos de diseño, coloración y escamación (Ortiz 1980) y aloenzimáticos (Victoriano et al. 2003). Sin embargo, estudios cromosómicos (Northland et al. 1987), 
reproductivos (Goldberg \& Rodriguez 1986) y osteológicos (Vidal \& Ortiz, 2004a) no encontraron ninguna diferencia sustancial. Análisis filogenéticos basados en secuencias de DNA encontraron que $M$. atacamensis, $M$. quadrivittatus y $M$. theresioides no son monofiléticos (Benavides et al. 2007), por lo que merece un estudio más profundo sobre su estatus taxonómico.

H. gaudichaudii (Duméril \& Bibron, 1836) es un pequeño reptil de la Familia Phyllodactylidae. En 1835, Gaudichaud capturó un ejemplar en Coquimbo (su terra typica), el que fue usado por Duméril \& Bibron (1836) para describir la especie (Capurro \& Codoceo 1950). Su distribución es por la costa de la IV Región hasta la Región de Antofagasta, al norte de Taltal (Capurro 1957). Según este último autor, Plate colectó 17 especímenes en la isla que se encuentra dentro del puerto de Totoralillo, los que fueron estudiados por Werner bajo el nombre de Gonadotes gaudichaudi. Este gecko es de color café grisáceo, con bandas transversales oscuras en su dorso (Donoso-Barros 1966). Se le encuentra oculto bajo las piedras de la zona litoral y supralitoral (Capurro, 1957), es de hábitos nocturnos, y muy sensible a las alzas de temperaturas (Donoso-Barros 1966). La historia taxonómica de esta especie ha sido confusa en lo relacionado a su estatus genérico, siendo descrita inicialmente como Gymnodactylus (Duméril \& Bibron 1836), Gonadotes (Lataste 1891) y Garthia (Donoso-Barros \& Vanzolini 1965). Estos últimos autores afirman que Garthia derivaría de Homonota, estando este género restringido a la vertiente oriental de Los Andes. En la Región de Atacama ha sido colectado precisamente en zonas litorales y supralitorales, aunque también en sectores interiores como Diego de Almagro (Núñez et al. 2001). Moreno et al. (2002) menciona la colecta de ejemplares capturados en el Parque Nacional Llanos de Challe, al norte de Huasco. Desconocemos la existencia de ejemplares en una serie tipo.

C. maculatus (Gravenhorst 1838) es un lagarto de gran tamaño, cuello fuertemente plegado, cabeza piramidal, ancha en la base con hocico ligeramente prolongado. Pertenece a la Familia Teiidae. Según Donoso-Barros (1960a) existen tres subespecies que se distribuyen desde Paposo, Región de Antofagasta, hasta Cauquenes, Región del Maule, estando las tres presentes en la Región de Atacama. La diferencia entre estas subespecies son, C. $m$. maculatus presenta cuatro manchas dorsales anilladas de blanco, desde el cuello a la cola, habita desde Copiapó hasta Cauquenes por el valle central, muy ligado a matorrales. En las costas de la Región de Atacama es reemplazado por $C$. m. atacamensis, que presenta un tono general café lechoso en el dorso, con diseños de líneas irregulares hasta la parte media del dorso. Ausencia de manchas negras en la parte anterior de la espalda. Su terra typica es la costa pedregosa de Caldera, cerca de las rompientes. C. m. manni presenta líneas de manchas negras paravertebrales muy pequeñas y sin anillos blancos. Dorso café oliváceo. Es menos robusto que C. m. maculatus, con mejillas poco prominentes. Se distribuye al sur de la Región de Antofagasta y su terra typica es Paposo. Moreno et al. (2001) mencionan a cuatro ejemplares capturados en Algarrobal, Copiapó, un espécimen en Chañaral y uno de El Faro, Caldera. La taxonomía de esta especie ha sido controversial. En primer término, Veloso et al. (2000) fijaron el neotipo para C. maculatus, ejemplar que se encuentra en el Museo Nacional de Historia Natural de Chile $\mathrm{N}^{\circ}$ 2909, acto que fue seguido por una compleja controversia taxonómica (para detalles ver Etheridge \& Savage 2006).

La terra typica, sus coordenadas y la ubicación de los especímenes tipo se resumen en la Tabla 2, y los puntos de recolecta determinados en colecciones taxonómicas y publicaciones científicas en la Figura 1.

\section{Estado de CONSERVACiÓN Y NIVELES DE ENDEMiSMO DE LOS} LAgARTOS DE ATACAMA.

El estado de conservación de los lagartos de Chile está definido por el Servicio Agrícola y Ganadero (SAG) en el Reglamento de la Ley de Caza D.S N5/1998 con modificaciones introducidas por el D.S N`53/2004 (Tabla 3). De las especies descritas para Atacama, dos están Fuera de Peligro (7,7\%), nueve son consideradas Raras $(34,6 \%)$, cinco Vulnerables (19,2\%); y una En Peligro (3,8\%). En dicho documento no se mencionan a las especies $L$. andinus, L. melanopleurus, L. nigroventrolateralis y $L$. robertoi que corresponden al $15,4 \%$ de las especies descritas para atacama. En relación a $L$. josephorum y $L$. manueli efectivamente están en el listado de especies, pero sin información sobre su estado de conservación. Para $L$. lorenzmulleri, L. nitidus y L. zapallarensis, el estado de conservación es Vulnerable, pero considerando solo las poblaciones de la zona central, sin dar información sobre las poblaciones de la zona norte.

En relación a los niveles de endemismos para la Región de Atacama (Tabla 3), y sin considerar a L. eleodori, $L$. lorenzmulleri, L. robertoi, y L. zapallarensis por no tener registros en colecciones taxonómicas de su presencia en Atacama, podemos afirmar que el $50 \%$ de las especies son endémicas de Atacama. El 45\% de las especies que habitan la región de Atacama, además, están presentes en otras regiones de Chile, y sólo dos especies tienen distribución en Argentina (L. andinus y L. nigriceps). 


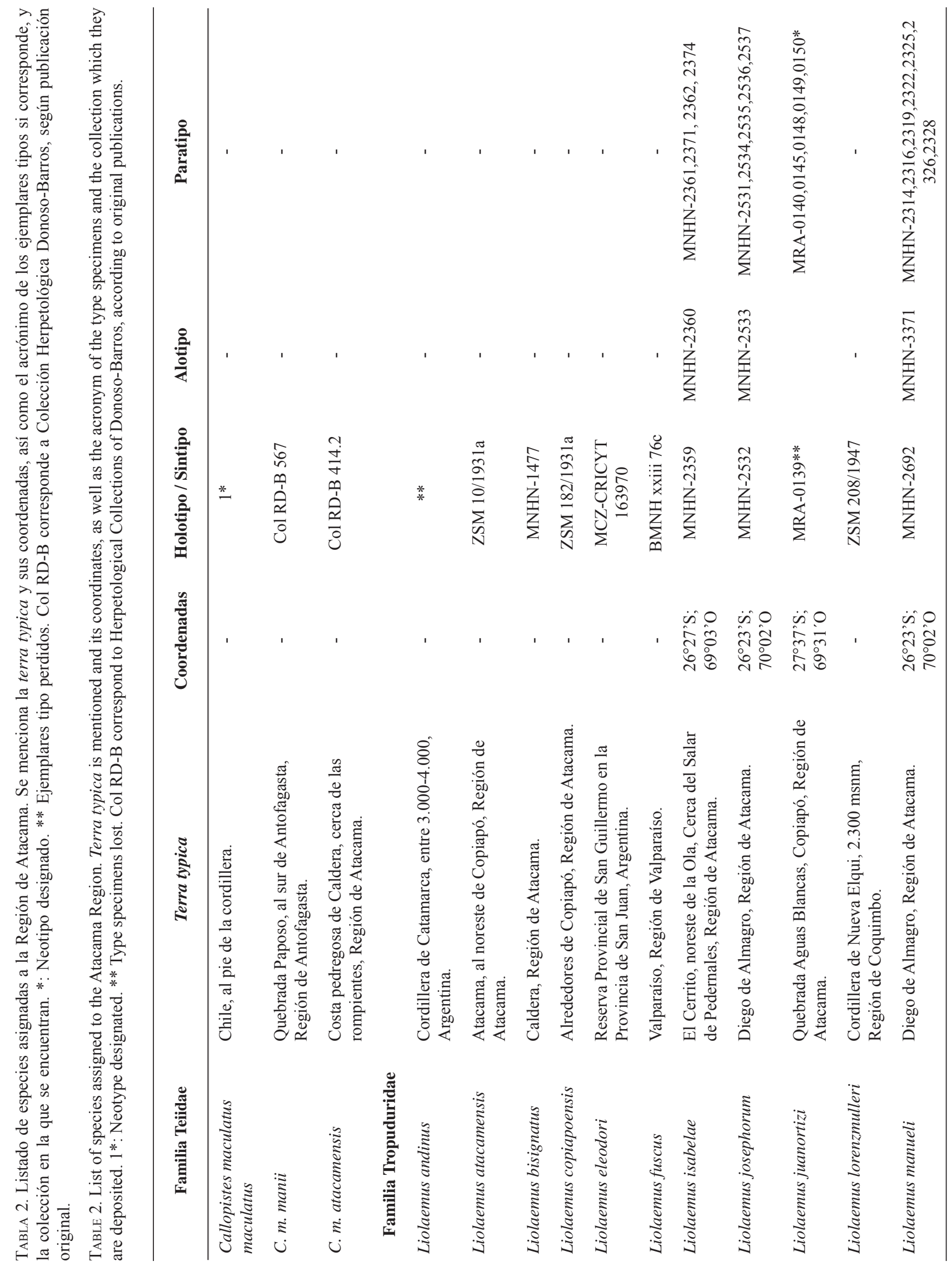




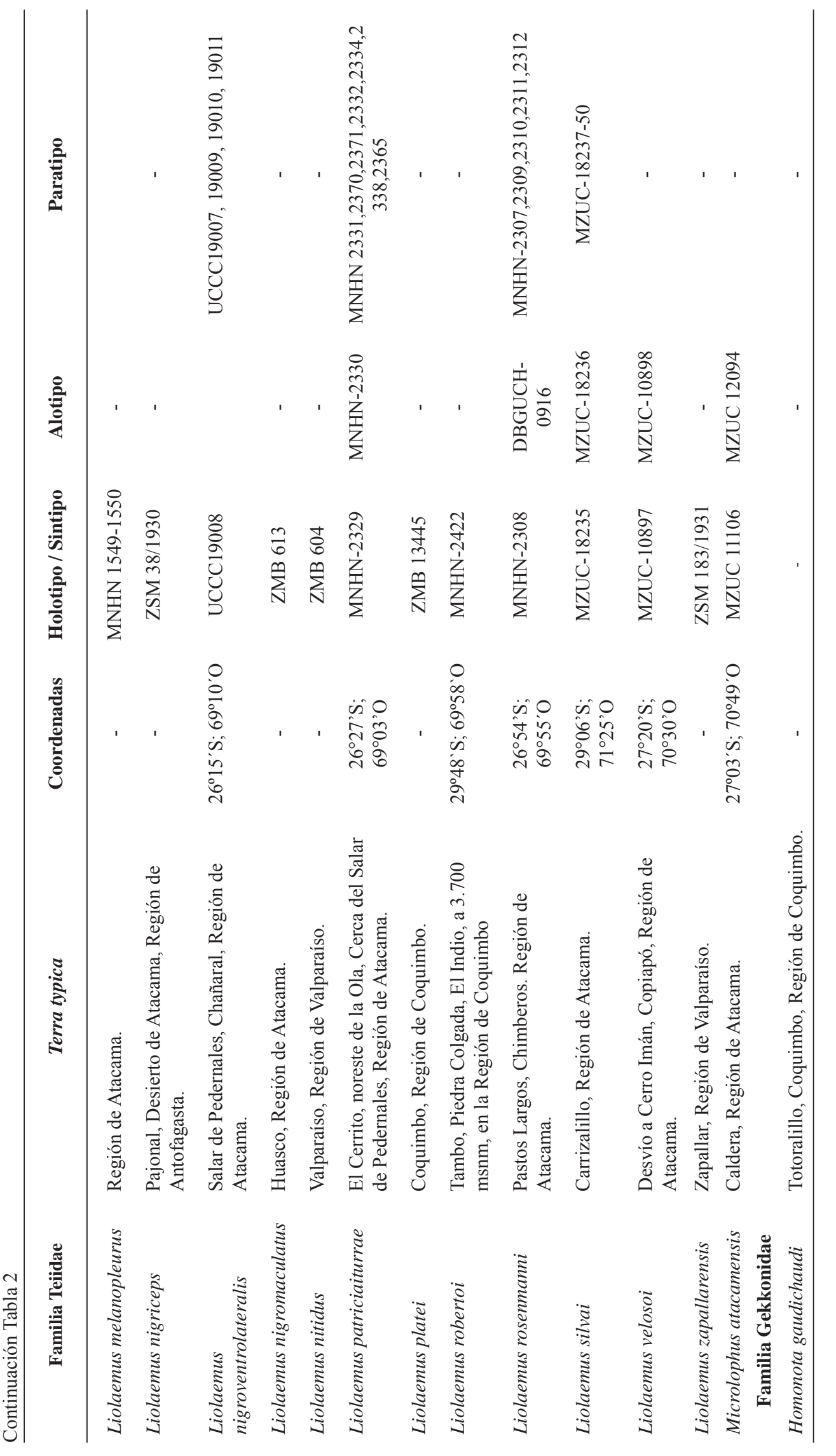


TABla 3. Estado de Conservación de los lagartos atacameños (SAG 2004) y nivel de endemismo.

TABLE 3. Conservation status of Atamana lizards (SAG 2004) and their level of endemism.

\begin{tabular}{|c|c|c|}
\hline Especie & Estado de Conservación & Endemismo \\
\hline Liolaemus andinus & - & 3 \\
\hline Liolaemus atacamensis & Rara & 2 \\
\hline Liolaemus bisignatus & Rara & 1 \\
\hline Liolaemus copiapoensis & Fuera de Peligro & 1 \\
\hline Liolaemus eleodori & Rara & - \\
\hline Liolaemus fuscus & Fuera de Peligro & 2 \\
\hline Liolaemus isabelae & Rara & 1 \\
\hline Liolaemus josephorum & Sin información & 1 \\
\hline Liolaemus juanortizi & En Peligro & 1 \\
\hline Liolaemus lorenzmulleri & Vulnerable & - \\
\hline Liolaemus melanopleurus & - & 1 \\
\hline Liolaemus manueli & Sin Información & 1 \\
\hline Liolaemus nigriceps & Vulnerable & 3 \\
\hline Liolaemus nigroventrolateralis & - & 1 \\
\hline Liolaemus nigromaculatus & Vulnerable & 2 \\
\hline Liolaemus nitidus & Vulnerable & 2 \\
\hline Liolaemus patriciaiturrae & Rara & 1 \\
\hline Liolaemus platei & Rara & 2 \\
\hline Liolaemus robertoi & - & - \\
\hline Liolaemus rosenmanni & Rara & 1 \\
\hline Liolaemus silvai & Vulnerable & 2 \\
\hline Liolaemus velosoi & Rara & 1 \\
\hline Liolaemus zapallarensis & Vulnerable & - \\
\hline Callopiste maculatus & Vulnerable & 2 \\
\hline Microlophus atacamensis & Vulnerable & 2 \\
\hline Homonota gaudichaudi & Rara & 2 \\
\hline
\end{tabular}

1: habita sólo la Región de Atacama, 2: habita en más de dos regiones, 3: especie con poblaciones fuera de Chile. 

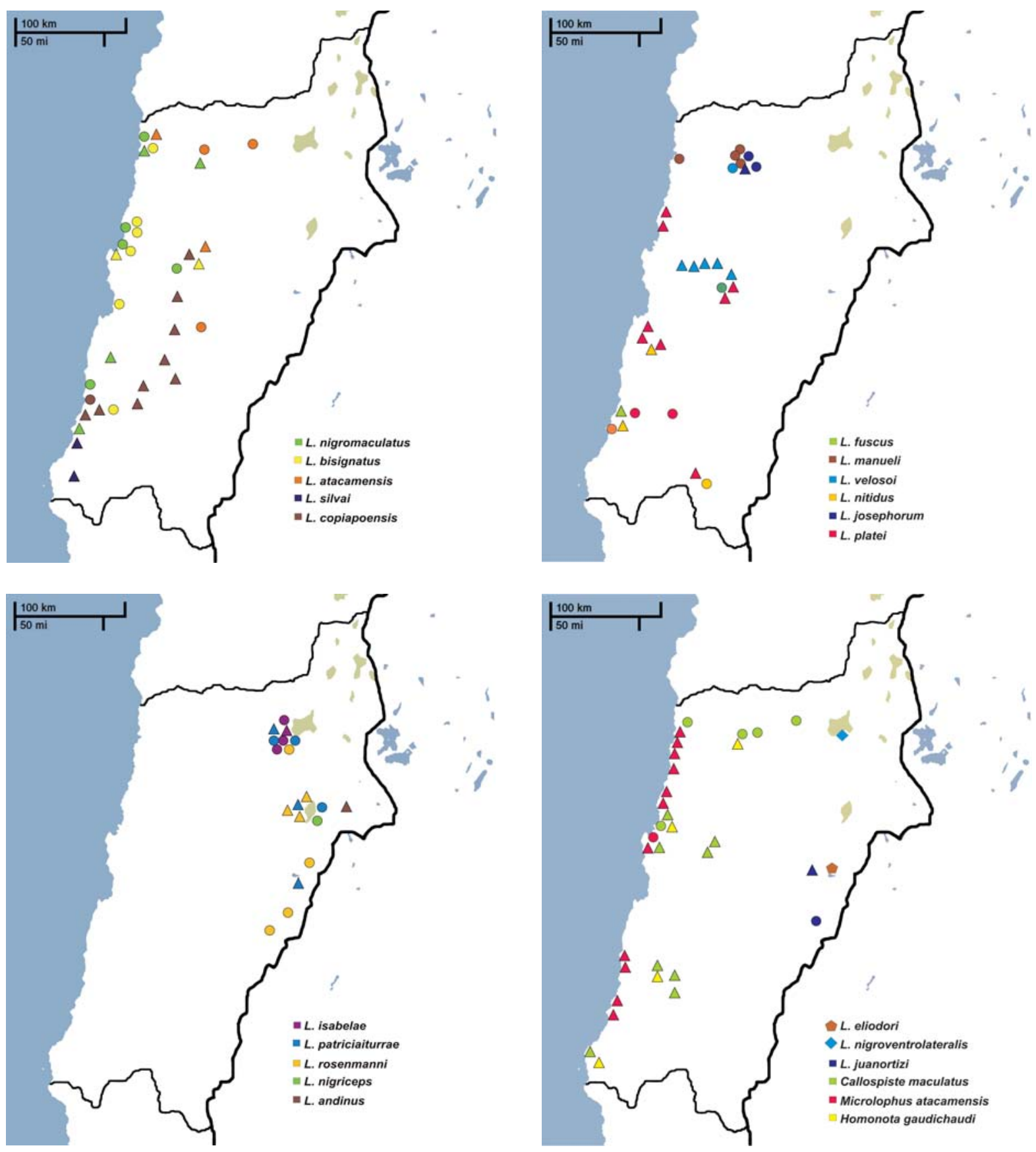

Figura 1. Mapas de distribución de las especies asignadas a la Región de Atacama. $\bullet$ datos de colecciones de museos nacionales (MNHN, UNAP y MZUC) y $\boldsymbol{\Delta}$ publicaciones científicas. Registros de captura basados en las siguientes publicaciones: Moreno et al. 2002.; Halloy et al. 1991, Vidal et al. 2002, Benavides et al. 2007; Donoso-Barros 1960a; Donoso-Barros 1960b; Fariña et al. 2008; Moreno et al. 2000; Navarro \& Núñez 1993; Núñez 1992; Núñez \& Torres-Mura 1992; Ortiz 1973; Ortiz 1977; Ortiz 1980; Ortiz 1981; Ortiz 1987; Ortiz 1989; Ortiz 1994; Vidal \& Ortiz 2003; Schulte II et al. 2004; Sepúlveda et al. 2008; Victoriano et al. 2003; Vidal \& Ortiz 2004; Young-Downey \& Moreno 1991; Moreno et al. 2001; Moreno \& Ibarra-Vidal 2004, Pincheira-Donoso \& Núñez 2005.

FIGURE 1. Distribution maps of the especies assigned to Atacama Region. $\bullet$ data collections from national museums (MNHN y UNAP) and $\Delta$ scientific papers. Catch records based on the following papers: Moreno et al. 2002.; Halloy et al. 1991, Vidal et al. 2002, Benavides et al. 2007; Donoso-Barros 1960a; Donoso-Barros 1960b; Fariña et al. 2008; Moreno et al 2000; Navarro \& Núñez 1993; Núñez 1992; Núñez \& Torres-Mura 1992; Ortiz 1973; Ortiz 1977; Ortiz 1980; Ortiz 1981; Ortiz 1987; Ortiz 1989; Ortiz 1994; Vidal \& Ortiz 2003; Schulte II et al. 2004; Sepúlveda et al. 2008; Victoriano et al. 2003; Vidal \& Ortiz 2004; Young-Downey \& Moreno 1991; Moreno et al. 2001; Moreno \& Ibarra-Vidal 2004, Pincheira-Donoso \& Núñez 2005. 


\section{DISCUSIÓN}

A partir de la información recopilada surgen algunas líneas de discusión que, de nuestra perspectiva, van desde problemas taxonómicos, de distribución, abundancia, riqueza de especies por áreas específicas, ecología y conservación.

Desde la perspectiva de la taxonomía existen varias problemáticas que merecen especial atención. En primer lugar se optó por asumir las propuestas nomenclaturales de Schulte et al. (2000) y Lobos et al. (2010), por ser realizadas en base a principios filogenéticos, aunque cabe mencionar que aún se requiere un análisis en base a más datos moleculares con que contrastar los análisis basados en morfología.

Por otro lado, la presencia de $L$. andinus en Chile es controversial. Pincheira-Donoso \& Núñez (2005) afirman que L. signifer (Koslowsky 1898), L. schmidti (Marx 1960), L. multiformis (Donoso Barros 1970), L. poecilochromus (Laurent 1982) y L. molinai (Valladares et al. 2002) son sinónimos de L. andinus, lo que ampliaría considerablemente su rango de distribución. Sin embargo, existen dudas sobre la interpretación taxonómica de estos autores, ya que no dan ninguna argumentación para esta sinonimización (Etheridge \& Frost 2010), más bien es una mera opinión que genera una mayor confusión en la taxonomía de los lagartos altoandinos, situación que es ampliamente discutida por Lobos et al. (2010). Los sintipos de L. andinus se encuentran perdidos (Etheridge \& Frost, 2010), por lo que la ausencia de este material hace dudar de cualquier interpretación de su estatus taxonómico y de las posibles sinonimizaciones que se han realizado, más aún cuando la terra typica es tan amplia y la descripción original de Koslowsky (1895) es breve e imprecisa. Por lo mismo, Halloy et al. (1991) afirma que es necesario hacer estudios más profundos en Catamarca, ya que al parecer dicha especie no habitaría en esa localidad y que su procedencia podría venir de otra parte de los Andes. Efectivamente, Halloy et al. (1991) asigna especímenes capturados al oriente del Salar de Maricunga a $L$. andinus. Por otro lado, especímenes capturados en la Laguna del Negro Francisco han sido asignados a $L$. eleodori (Núñez \& Torres-Mura 1992) y posteriormente determinados como L. rosenmanni (Pincheira-Donoso \& Núñez 2005), argumentando que correspondían a machos de esta última especie. Si Halloy et al. (1991) está en lo correcto, y los especímenes que se encuentran al oriente del Salar de Maricunga correspondieran a L. andinus y por otra parte, Pincheira-Donoso \& Núñez (2005) consideran que los especímenes que habitan a los alrededores de la Laguna del Negro Francisco fueran correctamente asignados a L. rosenmanni, entonces debe evaluarse la hipótesis que rosenmanni sea sinónimo de andinus, teniendo prioridad este último nombre (Robert Langstroth in litt.). Por otro lado, es importante considerar que el nivel de polimorfismo de las especies del grupo "montanus" (sensu Etheridge, 1995 y Lobos et al. 2010) es muy amplio, tanto a nivel ontogenético como en dimorfismo sexual (Valladares et al. 2002), lo que hace estrictamente necesario contar con herramientas moleculares para una mejor determinación de las especies de este grupo. Además, es fundamental contar con una extensa colección de ejemplares en un amplio rango de distribución, y analizar cuáles de ellos coinciden con los caracteres propuestos originalmente.

Otra especie dudosa para la Región de Atacama es $L$. nigriceps. Esta especie se menciona para la región en la zona de Aldebarán, en el río La Gallina, al este de Copiapó (Pincheira-Donoso \& Núñez 2005), cerca de $250 \mathrm{~km}$ más al sur de donde fue descrito originalmente. Philippi (1860) y Donoso Barros (1966) mencionan que el carácter diagnóstico que diferencia esta especie de la mayor parte de especies del grupo "montanus" que habitan la región es el capuchón negro que llega hasta los hombros. Algunos individuos que se han recolectado a unos $7 \mathrm{~km}$ al NE del Río La Gallina presentan dicho capuchón, pero siguen el patrón morfológico de los anteriormente asignados a L. rosenmanni. Al revisar los ejemplares de la colección de la Universidad de Concepción (MZUC 33501 - 33508), asignados a L. nigriceps, podemos constatar una gran similitud con los colectados en el Volcán Llullaillaco (MNHN 2971), básicamente en el número de escamas alrededor del cuerpo (86 - 106 para las de MZUC y $97-110$ para MNHN), forma y tipo de escamas dorsales (ambas con escamas pequeñas, redondeadas, lisas y yuxtapuestas), en el número de ciliares en ambos párpados, longitud de subocular, una corrida de lorilabiales en ambas poblaciones, pliegues laterocervicales, sin escudos prominentes en el borde anterior del meato auditivo, entre otras, pero que difieren en las longitudes corporales, como largo del cuerpo (para MZUC X $=80,4 \mathrm{~mm}$, rango $61-92,8$ $\mathrm{mm}$; MNHN X $=87,6 \mathrm{~mm}$, rango $84-90,1 \mathrm{~mm}$ ) y longitud axila - ingle (para MZUC X $=38,9 \mathrm{~mm}$, rango 29,8 - 34,6 $\mathrm{mm}$; MNHN X $=45,5 \mathrm{~mm}$, rango 44 - 46,9mm). También se observan diferencias en escudos cefálicos, como el tamaño de rostral, número y formas de postfrontales, entre otras. Sin embargo, estas diferencias podrían deberse sólo a una variación geográfica, y por lo tanto sería importante evaluar las divergencias genéticas entre ellas, dado que no se han identificado poblaciones intermedias.

Las especies que merecen especial atención son los llamados del grupo "nigromaculatus" (sensu Lobos et al. 2010). En este grupo se han reconocido catorce especies, de las cuales siete han sido registradas para Atacama. Sin embargo, al revisar los ejemplares de colecciones taxonómicas, hemos podido constatar que la variación morfológica que hay entre las especies de este grupo es muy alta, particularmente a las asignadas a $L$. bisignatus - L. copiapoensis y $L$. 
atacamensis - L. nigromaculatus, lo que ha traído como consecuencia que no exista consenso para determinarlas. Por un lado, L. copiapoensis ha sido sinonimizada a L. bisignatus (Pincheira-Donoso \& Núñez 2005). Sin embargo, Etheridge \& Frost (2010) reconocen esta especie afirmando que los argumentos dados por estos autores no son lo suficientemente sólidos para su sinonimización. Otro punto de controversia son las especies $L$. atacamensis y $L$. nigromaculatus. Para Pincheira-Donoso \& Núñez (2005) la forma de la mancha prehumeral es diagnóstica para estas especies, así como dos bandas dorsolaterales blancas que diferencian a L. atacamensis de L. nigromaculatus, sin embargo estos caracteres son muy polimórficas entre las distintas poblaciones. La dificultad en la determinación de las especies de este grupo se puede apreciar en la Figura 1, mapa que muestra las diversas localidades de captura para las distintas poblaciones asignadas a este grupo. Por ejemplo, hay especímenes asignados a L. copiapoensis para el norte de Huasco (MZUC 33792, 33797, 33821, 33823 ), zona cercana a la asignada como terra typica de $L$. nigromaculatus. Simonetti \& Núñez (1986) consideraron haber encontrado individuos de estas dos especies sintópicamente en la localidad de Sierra Las Tapias, $30 \mathrm{~km}$ al norte de Chañaral, las que pudieron ser diferenciadas por el patrón de coloración, número de escamas alrededor del cuerpo y caracteres morfométricos. Hasta la fecha no existen estudios con herramientas moleculares que permitan determinar inequívocamente sus grupos taxonómicos, ya que la búsqueda de caracteres diagnósticos ha sido infructuosa. Es altamente posible que con metodologías modernas se pueda esclarecer la situación taxonómica de este grupo que presenta una gran distribución en la Región de Atacama, habitando desde la costa hasta los $2.000 \mathrm{~m}$ sobre el nivel del mar.

A partir de la revisión de los ejemplares tipo de L. silvai, podemos concluir que es una buena especie, claramente asignada al grupo "nigromaculatus" (sensu Lobos et al. 2010), caracterizándose básicamente por su fuerte melanismo ventral en los machos. Sin embargo, las hembras y juveniles analizados tienen importantes similitudes con el resto de las especies de este complejo, lo que dificulta su diferenciación.

L. platei presenta un amplio rango de distribución (DíazPáez et al. 2008, Ortiz 1973), con un morfotipo que se mantiene a lo largo de su rango de distribución, pero con importantes variaciones en su patrón de coloración. Un análisis filogenético realizado entre poblaciones de esta especie determinó que las diferencias entre la población de Totoralillo (Región de Coquimbo, IV Región y que corresponde a su terra typica) y Pan de Azúcar (extremo norte de la Región de Atacama), es la misma que entre $L$. platei y L. josephorum, situación que nos permite cuestionar el estatus taxonómico de las poblaciones extremas de L. platei. Por otro lado, aún no se esclarece la relación filogenética de L. platei con $L$. velosoi, así como entre $L$. velosoi y $L$. josephorum para argumentar la sinonimización entre ellas. En este grupo, especial atención requerirá la revisión de platei con melanopleurus, pues el número de escamas alrededor del cuerpo parece no ser suficiente para diagnosticarlas.

Desde la descripción de las subespecies de Callopiste maculatus por Donoso-Barros (1960a) que no se ha revisado la taxonomía y distribución de dichas formas. No está claramente establecido si C. m. manni alcanza la Región de Atacama, y las diferencias reconocidas por DonosoBarros (1960a, 1966) para estas subespecies parecen no ser lo suficientemente claras, ya que, por ejemplo, las manchas dorsales anilladas de blanco que diagnostican a $C$. m. maculatus son frecuentes en las poblaciones costeras de Caldera, lugar donde habita C. $m$ atacamensis( CVZA 0148 y 0149). Además, el tono dorsal lechoso que se señala como diagnóstico para la subespecie atacamensis es muy variable, y depende del estado de desarrollo y sexo del individuo, ya que hemos observado especímenes macho con un color rojo ladrillo muy fuerte en las poblaciones de Caldera comparadas con las de Copiapó (CVZA 0200). Se requiere de análisis más exhaustivos sobre la morfología y genética de las distintas poblaciones de esta especie.

A pesar que la mayor parte de los lagartos atacameños presentan especímenes tipo, aún falta designar especímenes neotipo para algunas especies en que se han extraviado sus ejemplares tipos, como por ejemplo L. juanortizi y L. andinus. El objetivo es lograr la mayor estabilidad nomenclatural posible para evitar confusiones taxonómicas y los listados de especies sean permanentes y estables.

Además de la reevaluación taxonómica de varias especies atacameñas, es imperativo evaluar sus rangos de distribución. Se ha documentado, por ejemplo, la colecta de $L$. fuscus en la localidad de Huasco (Moreno et al. 2001), especie que es común en la zona central de Chile y que alcanza la Región de Coquimbo. Esta distribución puede ser posible considerando que el valle de Huasco tiene una rica vegetación aledaña al Río Huasco, y que podría corresponder al límite norte de muchas especies de lagartos comunes en la zona central de Chile, como L. nitidus. Sin embargo, no hemos tenido la posibilidad de conocer dichos especímenes, y después de recorrer las costas de la Provincia de Huasco, aún no hemos podido constatar su presencia en Atacama. En este mismo sentido, cabe reevaluar la presencia de L. lorenzmuelleri, L. zapallarensis, L. robertoi y L. eleodori en Atacama, que son especies mencionadas para la región por diversos autores (Díaz-Páez et al. 2008, Núñez \& Torres-Mura 1992, Donoso-Barros 1966), pero no existen registros de captura 
de estas especies en las colecciones taxonómicas y en las publicaciones analizadas.

La información disponible de la ecología de los lagartos en la región es muy limitada y está casi particularmente restringida a M. atacamensis (Fariña et al. 2008, Sepúlveda et al. 2008, Vidal et al. 2002) y en segundo lugar a C. maculatus (Vidal \& Ortiz 2004b). Sin embargo, hay menciones generales sobre ciertos ecotipos y de las especies asociadas a ellas (Donoso-Barros 1960b). No hay estudios ecológicos sobre los factores que determinen la distribución y la abundancia de los lagartos atacameños, y particularmente en el género Liolaemus. Solo Pincheira-Donoso (2009) analiza las adaptaciones ecológicas de $L$. platei y $L$. velosoi, entre otras especies extrarregionales.

La falta de información, tanto en la taxonomía, como en la distribución y abundancia de las especies regionales, no permite tener clara certeza en el nivel de endemismo de las mismas. Veloso et al. (1995) mencionan diez especies de saurios para Atacama, con cuatro de ellas endémicas, siendo una región con una intensidad de exploración regular. En Vidal et al. (2009) se analiza los patrones de distribución de las especies de reptiles y anfibios de Chile con la finalidad de evaluar centros de diversidad, rareza y endemismo, encontrando que la mayor riqueza de especies se encuentran a partir del paralelo $31^{\circ} \mathrm{S}$ al $44^{\circ} \mathrm{S}$. Este análisis está basado en datos de rangos de distribución encontrados en publicaciones y datos de campo. A la luz de los antecedentes analizados y evaluados en el presente trabajo, se carecería de la información necesaria para establecer los niveles de endemismos para la Región de Atacama, es decir el número total de especies en los cuadrantes señalados y el número de cuadrantes con cada una de las especies (Moreno et al. 2006). Consideramos necesario reevaluar los niveles de endemismo de las especies de lagartos en la Región de Atacama, a la luz de más información que permita una mayor certeza de la riqueza de especies y sus rangos de distribución. Esta falta de información también incide en no poder determinar con claridad los estados de conservación de las especies de lagartos atacameños, lo que se hace fundamental desarrollar, dado el alto nivel de explotación de recursos naturales que existe en esta región, particularmente en el área de la minería.

\section{AGRADECIMIENTOS}

Agradezco la colaboración de Andrés Vejar, Asistente del CRIDESAT, en la recopilación de información, preparación de figuras y revisión del manuscrito. Al Dr. Juan Carlos Ortiz por permitirme revisar los lagartos de las Colecciones Cientificas de la Universidad de Concepción (UCCCMZUC). Al profesor Herman Núñez por permitirme revisar los lagartos del Museo de Historia Natural años atrás y que dicha información aún es útil para el trabajo de la Herpetología atacameña. A los Drs. Cristián Abdala y Robert Langstroth por compartir conmigo sus apreciaciones sobre la especie Liolaemus andinus y otras relacionadas; algunas de aquéllas son mencionadas en este manuscrito. A la Dra. Marcela Vidal por enviarme artículos relacionados a los lagartos de Atacama. Este manuscrito es parte del Programa de Levantamiento de Información de la Biodiversidad de Atacama, organizado y ejecutado por el Centro Regional de Investigación y Desarrollo Sustentable de Atacama (CRIDESAT).

\section{BIBLIOGRAFÍA}

Benavides, B., Baum, R., McClellan, D. \& Sites, J.W. JR. 2007. Molecular phylogenetics of the lizard genus Microlophus (Squamata:Tropiduridae): aligning and retrieving indel signal from nuclear introns. Systematic Biology 56(5): 776-797.

Boulenger, G.A. 1885. Catalogue of the lizards in the British Museum (Natural History), London. 497 pp.

Burt, C.E. \& Burt, M.D. 1930. The South American lizards in the collection of the United States National Museum. Proceedings of the United States National Museum 78: 152.

CAPURro, L.F. 1957. Distribución de Gonatodes gaudichaudi (Duméril \& Bibron) en Chile. Investigaciones Zoológicas Chilenas 3: 141-142.

Capurro, L.F. \& Codoceo, M. 1950. Gonatodes gaudichaudi (Duméril \& Bibron). Investigaciones Zoológicas Chilenas 1: $15-16$

Cortés A, Torres-Mura, J.C., Contreras, L. \& Pino, C. 1995. Fauna de vertebrados de los Andes de Coquimbo: Cordillera de Doña Ana. Ediciones Universidad de La Serena, La Serena, Chile. viii +96 pp.

Díaz-PÁez, H., NúÑez, J., NúÑEz, H. \& Ortiz J.C. 2008. Estado de conservación de anfibios y reptiles. En: Herpetología de Chile (Eds. Vidal, M. \& M. Labra), pp. 233-267. Science Verlg. Santiago.

Donoso-Barros, R. 1960a. La familia Teiidae en Chile. Revista Chilena de Historia Natural 55: 41-54.

Donoso-Barros, R. 1960b Ecología de los reptiles chilenos. Investigaciones Zoológicas Chilenas 6: 65-72.

Donoso-Barros, R. 1960c. Estado actual del género Tropidurus en chile. Sesiones de Biología de la Universidad del Cuyo. pp 27-29. Mendoza.

Donoso - Barros, R. 1966. Reptiles de Chile. Ediciones de la Universidad de Chile. Santiago. 458 pp.

Donoso - BArros, R.1970. Catálogo herpetológico chileno. Boletín del Museo Nacional Historia Natural de Chile 31: 49-124.

Donoso - Barros, R.1974. Nuevos reptiles y anfibios de Chile. Boletín de la Sociedad de Biología de Concepción 48: 217229.

Donoso-Barros, R. \& Vanzolini, P.E. 1965. El género Garthia y los geckos afines. Publicación Ocasional del Museo de Historia Natural 7: 1-8.

Duméril, A.M.C. \& Bibron, G. 1836. Erpétologie générale ou histoire naturelle complète des reptiles, Edicion Roret, Paris: 3, 413 pp. 
Duméril, A.M.C. \& Bibron, G. 1837. Erpétologie générale ou histoire naturelle complète des reptiles, Edicion Roret, Paris: 4, $571 \mathrm{pp}$.

Elgueta, E., Reid, S., Pliscoff, P., Méndez, M.A., Núñez J. \& Smith-Ramírez, C. 2006. Catastro de vertebrados terrestres y análisis en seis hábitats presentes en la reserva nacional Futaleufu, Provincia de Palena, X Región, Chile. Gayana 70 (2): 195-205.

Etheridge, R. 1995. Redescription of Ctenoblepharys adspersa (Tschudi, 1845), and the taxonomy of Liolaeminae (Reptilia: Squamata: Tropiduridae). American Museum Novitates. 3142: 1-34.

Etheridge, R \& EspinOZA, R.E. 2000. Taxonomy of the Liolaeminae (Squamata: Iguania: Tropiduridae) and a semiannotated bibliography. Smithsonian Herpetological Information Service, 126, 1-64.

Etheridge, R \& Frost, D. 2010. Liolaemidae. Citing Electronic Catalogue. American Museum of Natural History. URL: http://research.amnh.org/vz/herpetology/f/Liolaemidae.pdf

Etheridge, R. \& Savage, J.M. 2006. Stability restored to the name Phymaturus palluma: response to Cei and Scolaro (2006). Herpetological Review 37:406-407.

Fariña, J. M., Sepúlveda, M., Reyna, M. V., Wallem, K. P. \& Ossa-Zazzali, P. G. 2008. Geographical variation in the use of intertidal rocky shores by the lizard Microlophus atacamensis in relation to changes in terrestrial productivity along the Atacama Desert coast. Journal of Animal Ecology 77: 458-468

Frost, D.R. 1992. Phylogenetic analysis and taxonomy of the Tropidurus group of lizards (Iguania: Tropiduridae). American Museum Novitates 3033, 1-68.

Gay. C. 1848. Historia física y política de Chile. Tomo segundo, Zoología. Casa del Autor, París. Museo Nacional de Historia Natural, Santiago.

Gigoux, E.E. 1927. El Leiolaemus nigromaculatus. Revista Chilena de Historia Natural 31(1): $182-185$

Goldberg, S.R. \& Rodríguez, E. 1986. Reproductive cycles of two iguanid lizards from northern Chile, Tropidurus quadrivittatus and Tropidurus theresioides. Journal of Arid Environments 10: 147-151.

Halloy, M., Grosse, C. \& Laurent, R.F. 1991. Liolaemus andinus (Iguanidae) de deux côtés des Andes. Revue Français de Aquariologie 18: 61-64.

Hellmich, W. 1952. Contribución al conocimiento de la sistemática y evolución del género Liolaemus. Investigaciones Zoológicas Chilenas 1: 7-15.

KosLowsky, J. 1895. Batracios y reptiles de La Rioja y Catamarca (República Argentina) recogidos durante los meses de febrero a mayo de 1895 (expedición del director del Museo). Revista Museo La Plata 6: 357-370.

LAURENT, R.F. 1992. On some overlooked species of the genus Liolaemus Wiegmann (Reptilia-Tropiduridae) from Peru. Breviora 494: 1-33.

Lово, F. 2005. Las relaciones filogenéticas dentro del grupo chiliensis (Iguania: Liolaemidae: Liolaemus): sumando nuevos caracteres y taxones. Acta Zoológica Lilloana 49: 67-89.

Lobo, F., Espinoza, R. \& S. Quinteros. 2010. A critical review and systematic discussion of recent clasification proposal for liolaemid lizards. Zootaxa 2549: 1-30.

Myers N., R.A. Mittermeir, C.G. Mittermeir, G.A. Fonseca \& J. KENT. 2000. Biodiversity hotspots for conservation priorities. Nature 403: 853-858.

Moreno, R.A., Hernández, C.E., Rivadeneira, M.M., Vidal, M.A. \& Rozbaczylo, N. 2006. Patterns of endemism in southeastern Pacific benthic polychaetes of the Chilean coast. Journal of Biogeography 33: 750-759.

Moreno R, Moreno, J., Torres-Pèrez, F. \& Ortiz, J.C. 2000. Reptiles del Parque Nacional "Nevados de Tres Cruces" (III Región, Chile). Boletín Sociedad Biología de Concepción, Chile 71: 41-43.

Moreno, R., Moreno, J., Torres - Pérez, F., Ortiz, J.C. \& Breskovic, A. 2001. Catálogo herpetológico del Museo del Mar de la Universidad Arturo Prat de Iquique, Chile. Gayana 65: 149-153.

Moreno, R., Moreno, J., Ortiz, J.C., Victoriano, P. \& Torres Pérez, F. 2002. Herpetofauna del Parque Nacional Llanos de Challe (III Región, Chile). Gayana 66: 7-10.

Müller, L. \& Hellmich, W. 1933. Beiträge zur Kenntnis der Herpetofauna Chiles. VII. Der Rassenkreis des Liolaemus nigromaculatus. Zoologischer Anzeiger 103: 128-142.

NAVArro, J. \& NúÑEZ, H. 1993. Liolaemus patriciaiturrae y Liolaemus isabelae, dos nuevas especies de lagartijas para el norte de Chile. Aspectos biogeográficos y citotaxonómicos. Boletín del Museo Nacional de Historia Natural de Chile. 44: 99-113.

Northland, I., Capetillo, J., Iturra, P. \& Veloso, A. 1987. Estudios morfológicos y cromosómicos en el género Tropidurus (Iguanidae) del norte de Chile. Anales del Museo de Historia Natural de Chile 18: 115-122.

NúÑEZ, H. \& JAKSIC, F. 1992. Lista comentada de los reptiles terrestres de Chile continental. Boletín del Museo Nacional de Historia Natural de Chile 43: 73-91.

NúÑEz, H., Navarro, J., Sufan, J. \& Galaz, J.L.1998. Distribución geográfica de Phrynosaura (Reptilia: Tropiduridae). Noticiario Mensual Museo Nacional de Historia Natural de Chile 334: 11-14.

NúÑez, H., Schulte II, J.A. \& Garín, C. 2001. Liolaemus josephorum, nueva especie de lagartija para el norte de Chile. Boletín del Museo Nacional de Historia Natural de Chile 50: 91-107.

NúÑEz, H. \& Torres - Mura, J.C. 1992. Adiciones a la herpetofauna de Chile. Noticiario Mensual del Museo Nacional de Historia Natural de Chile 322: 3-8.

Ortiz, J.C. 1973. Nota distribucional sobre el Liolaemus platei platei Werner (Squamata: Iguanidae). Anales del Museo de Historia Natural de Valparaíso, Chile 6: 75-77.

Ortiz, J.C. 1977. Revisión taxonómica del género Tropidorus en Chile. I Reunión Iberoamericana de Zoología de Vertebrados, La Rábida, Venezuela, 355-377.

OrtiZ, J.C. 1980. Estudio comparativo de algunas poblaciones de Tropidurus de la costa chilena. Anales del Museo de Historia. Natural de Valparaíso, Chile. 13:267-279.

OrTIZ, J.C. 1981. Estudio multivariado de las especies de Liolaemus del grupo nigromaculatus (Squamata, Iguanidae). Anales del Museo de Historia Natural de Valparaíso 14: 247-265.

Ortiz, J.C. 1987. Une nouvelle espèce de Liolaemus (Sauria, Iguanidae) du Chili. Bulletin Museum National d'Histoire Naturelle, Paris, ser. 9(A): 265-270.

Ortiz, J.C. 1989. Description de Liolaemus silvai sp. nov. (Sauria, Iguanidae) du "Norte Chico" du Chili. Bulletin Museum National d'Histoire Naturelle, Paris, ser. 11(A): 247-252.

Ortiz, J.C. 1994. Una nueva especie de lagarto altoandino del género Liolaemus (Sauria, Tropiduridae). Boletín de la 
Sociedad de Biología de Concepción 65: 191-195.

Ortiz, J.C. 2008. Historia de la herpetología en Chile. En Herpetología de Chile (Eds. Vidal, M. \& M. Labra), pp. 3-29. Science Verlg. Santiago.

Ortiz, J.C. \& NúÑEz, H. 1986. Catálogo crítico de los tipos de reptiles conservados en el Museo Nacional de Historia Natural Santiago, Chile. Publicación Ocasional Museo Nacional de Historia Natural, Chile 43: 1-23.

OrtiZ, J.C. \& SerEy, I. 1979. Análisis factorial de correspondencias de las especies del género Tropidurus de Chile. Archivos de Biología y Medicina Experimentales, Chile 12: 203208.

PhilipPI, R.A. 1860. Reise durch die Wüste Atacama, auf Befehl der chilenischen Regierung in Sommer 1853-1854, Librería de Eduard Anton, Halle, 192 pp.

Pincheira-Donoso, D., Hodgson, D.J., Stipala, J. \& Tregenza, T. 2009. A phylogenetic analysis of sex-specific evolution of ecological morphology in Liolaemus lizards. Ecological Research 24: 1223-1231.

Pincheira-Donoso, D. \& NúÑEz, H. 2003. Liolaemus robertoi, una nueva especie de los Andes del norte de Chile perteneciente al grupo ruibali (Iguania: Tropiduridae: Liolaeminae). Multequina, Latin American Journal of Natural Resources 12: $1-15$

Pincheira-Donoso, D. \& NúÑEZ, H. 2005. Las especies chilenas del género Liolaemus Wiegmann. 1834 (Iguania: Tropiduridae: Liolaeminae). Taxonomía, sistemática y evolución. Publicación Ocasional, Museo Nacional de Historia Natural de Chile 59: 1-486.

Pincheira-Donoso, D. \& NúÑEz, H. 2007. Conspecificity of Liolaemus isabelae Navarro \& Núñez, 1993 and Liolaemus nigroventrolateralis Ortiz, 1994 (Iguania: Tropiduridae: Liolaeminae) from Northern Chile. Herpetological Journal $17,65-67$.

RAU J.R. 2005. Biodiversidad y colecciones científicas. Revista Chilena de Historia Natural 78: 341-342, 2005.

Schulte II, J.A., Losos, J.B., Cruz, F.B. \& NúÑEz, H. 2004. The relationship between morphology, escape behaviour and microhabitat occupation in the lizard clade Liolaemus (Iguanidae: Tropidurinae: Liolaemini). Journal of Evolutionary Biology 17: 408-420.

Schulte II J.A., Macey, J.R., Espinoza, R.E. \& Larson, A. 2000. Phylogenetic relationships in the iguanid lizard genus Liolaemus: multiple origins of viviparous reproduction and evidence for recurring Andean vicariance and dispersal. Biological Journal of the Linnean Society 69: 75-102.

Sepúlveda, M., Vidal M.A., Fariña, J.M. \& Sabat, P. 2008. Seasonal and geographic variation in thermal biology of the lizard Microlophus atacamensis (Squamata: Tropiduridae). Journal of Thermal Biology 33:141-148.

Servicio Agrícola y Ganadero (SAG). Reglamento de la Ley de Caza D.S N 5 / 1998 con modificaciones introducidas por el D.S N 53 / 2004.

Silva-ARÁnguIZ, E. 2001. Recopilación de la literatura herpetológica chilena desde 1780 hasta 2010. Centro de Investigación Avanzada en Ecología y Biodiversidad, Departamento de Ecología, Pontificia Universidad Católica de Chile. http:// www.bio.puc.cl/auco/artic07/herpeto1.htm Accesado: 28 de enero de 2010

Simonetti, J.A., Arroyo, M.T.K., Spotorno, A.E. \& LozadA, E. 1995. Diversidad biológica en Chile. Comisión Nacional de Ciencia y Tecnología, Santiago, Chile. 364 pp.

SimonetTi, J.A. 1999. Diversity and conservation of terrestrial vertebrates in mediterranean Chile. Revista Chilena de Historia Natural 72: 493-500.

SimonetTi, J.A. \& NúÑEz, H. 1986. Sympatry and Taxonomy of two lizards of the Liolaemus nigromaculatus group in northern Chile. Journal of Herpetology 20(3): 474-475.

Valladares, J. P. 2004. Nueva especie de lagarto del género Liolaemus (Reptilia: Liolaemidae) del norte de Chile, previamente confundido con Liolaemus (= Phrynosaura) reichei. Cuadernos de Herpetología 18: 41-51.

Valladares, J. P., Etheridge, R., Schulte II, J.A., Manríquez, G. \& Spotorno, A. 2002. Nueva especie de lagartija del norte de Chile, Liolaemus molinai (Reptilia: Liolaeminae). Revista Chilena de Historia Natural 75: 473-489.

Veloso, A. \& Navarro, J. 1988. Lista sistemática y distribución geográfica de anfibios y reptiles de Chile. Bollettino del Museo Regionale di Scienze Naturali di Torino. 6(2): 481539.

Veloso, A., Ortiz, J.C., Navarro, J., NúÑez, H., Espejo, P. \& Labra, M.A. 1995. Reptiles. En: Diversidad Biológica de Chile (Eds. Simonetti, J.A., Arroyo, M.K., Spotorno, A.E. \& Lozada, E.), pp. 326-335. Comisión Nacional de Ciencia y Tecnología, Santiago.

Veloso, A., NúÑEz H., \& CEI. J.M., 2000. Fixation and description of a neotype for Lacerta palluma Molina 1782 (currently) - Squamata: Teiidae. Bollettino del Museo Regionale di Scienze Naturali di Torino 17: 257-268.

Victoriano, P., Torres-Perez, F., Ortiz, J.C., Parra, L.E., Northland, I. \& CAPETILLO, J.2003. Variación aloenzimática y parentesco evolutivo en especies de Microlophus del grupo "peruvianus" (Squamata: Tropiduridae). Revista Chilena de Historia Natural 76: 65 - 78.

Vidal, M.A. \& Labra, A. 2008. Herpetología de Chile. Science Verlag, Santiago, Chile. XXIII + 593 pp.

Vidal, M. \& Ortíz, J.C. 2004. Análisis osteológico en dos especies de Microlophus (Sauria, Tropiduridae) de la costa chileno - peruana. Gayana 68: 9 - 19.

Vidal, M. \& Ortíz, J.C. 2004. Callopistes palluma. Cannibalism. Herpetological Review 34(4): 364 - 365.

VidAl, M.A., ITURRA - Cid, M. \& ORTIZ, J.C. 2008. Clasificación de anfibios y reptiles. En Herpetología de Chile (Eds. Vidal, M. \& M. Labra), pp. 195 - 231. Science Verlg. Santiago.

Vidal, M., Ortíz, J.C. \& Labra, A. 2002. Sexual and age differences in ecological variables of the lizard Microlophus atacamensis (Tropiduridae), from northern Chile. Revista Chilena de Historia Natural 75: 283 - 292.

Vidal, M., Soto, E. \& Veloso, A. 2009. Biogeography of Chilean herpetofauna: distributional patterns of species richness and endemism. Amphibia - Reptilia 30: 151 - 171.

Young-Downey, A. \& Moreno, J. 1991. A new species of tropidurine lizard (Squamata: Tropiduridae) from los Andes of northern Chile. Gayana Zoología, 55 (4): 391 - 396. 